\title{
LETTERS
}

\section{Reconciling complexity with stability in naturally assembling food webs}

\author{
Anje-Margriet Neutel ${ }^{1} \uparrow$, Johan A. P. Heesterbeek ${ }^{2}$, Johan van de Koppel ${ }^{3}$, Guido Hoenderboom ${ }^{4}$, An Vos $^{5}$, \\ Coen Kaldeway ${ }^{5}$, Frank Berendse ${ }^{6}$ \& Peter C. de Ruiter ${ }^{5,7}$
}

Understanding how complex food webs assemble through time is fundamental both for ecological theory and for the development of sustainable strategies of ecosystem conservation and restoration. The build-up of complexity in communities is theoretically difficult, because in random-pattern models complexity leads to instability ${ }^{1}$. There is growing evidence, however, that nonrandom patterns in the strengths of the interactions between predators and prey strongly enhance system stability ${ }^{2-4}$. Here we show how such patterns explain stability in naturally assembling communities. We present two series of below-ground food webs along natural productivity gradients in vegetation successions ${ }^{5,6}$. The complexity of the food webs increased along the gradients. The stability of the food webs was captured by measuring the weight of feedback loops ${ }^{7}$ of three interacting 'species' locked in omnivory. Low predator-prey biomass ratios in these omnivorous loops were shown to have a crucial role in preserving stability as productivity and complexity increased during succession. Our results show the build-up of foodweb complexity in natural productivity gradients and pin down the feedback loops that govern the stability of whole webs. They show that it is the heaviest three-link feedback loop in a network of predator-prey effects that limits its stability. Because the weight of these feedback loops is kept relatively low by the biomass build-up in the successional process, complexity does not lead to instability.

Ecologists have long studied processes of community assembly and have revealed general principles governing community diversity, dynamics and functioning in vegetation successions ${ }^{8}$, that is, on a single trophic level. The understanding of communities at multitrophic levels, however, is much less advanced. In processes of ecological succession, increasing productivity could give longer food chains $^{9-11}$, although opposite trends have also been found ${ }^{12}$, and it has been argued that stability constraints may limit food-chain length ${ }^{13}$. Moreover, the assembly of complex food webs is not selfevident, because random community models suggest that complexity promotes instability ${ }^{1}$. However, there has been increasing evidence that the nonrandom patterning of strong and weak links in food webs greatly enhances the stability of these networks ${ }^{2-4}$. It has been suggested that such stabilizing patterns can be caused by the decrease in biomass over increasing trophic levels, which means that long feedback loops in the food webs contain relatively weak links ${ }^{7}$. In a comparison between steady states of food-web models varying in complexity, and with hypothetical biomass pyramids, it was shown that complexity does not enhance instability ${ }^{7}$.

So far, there have been no studies that show and quantify the buildup of complexity of food webs in long-term successional processes.
The question still stands: do food webs become more complex as ecosystems develop and, if so, how can these systems exist-what keeps them stable, despite their increasing complexity?

Here we present a series of food webs sampled over two successional gradients along which natural productivity varies. We show that the food webs increase in complexity and that it is not their complexity as such, but the organization of link strengths over feedback loops, measured as 'maximum loop weight' of omnivorous loops ${ }^{7}$, that governs their stability. We do this within the classical framework of May ${ }^{1}$. Maximum loop weight has been shown to characterize and explain the natural organization of food webs by distinguishing empirically patterned webs from their randomized counterparts $^{7}$. However, so far the approach has not been used to quantify differences between various empirical food webs, nor to study the process of community assembly. This is what we will do here.

We studied two successions of below-ground food webs in sandy dune soils: one on the Waddensea Island of Schiermonnikoog in the north of the Netherlands ${ }^{5}$ and the other at Hulshorsterzand, on the Veluwe, in the central Netherlands ${ }^{6}$. The study sites, which constitute the two gradients, represent four consecutive stages in chronosequences of early primary vegetation succession, increasing in above-ground ${ }^{5,6}$ and below-ground productivity (see Methods). The observed soil organisms were categorized as 'functional groups' on the basis of taxonomy, life-history traits and diet ${ }^{14,15}$. We regarded observed yearly average biomass densities (see Methods and Supplementary Table 1) as steady states, that is, as stage-equilibria interpreted in the sense of ref. 9 .

We found an increase in complexity of the food webs along both gradients. Food-web diagrams representing the four successional stages illustrate this increase (Fig. 1). When we quantify this, we see that the number of trophic groups $(n)$, the maximum food-chain length and the link density $\left(n C\right.$, where $C$ is connectance $\left.{ }^{1,16}\right)$ all increased along the gradients (Supplementary Table 2). There was not a strict growth of the webs, because in the last stage phytophagous nematodes tended to disappear (Fig. 1).

We calculated the interaction strengths-effects of the trophic groups on each other-using the methods from refs. 2 and 17 (see Methods). We determined food-web stability (that is, the ability to return to the steady state after a small perturbation) using the method from ref. 7 (see Methods).

We examined how food-web structure is related to system stability. To do this, we first related stability to two previously proposed measures of food-web complexity that average over all

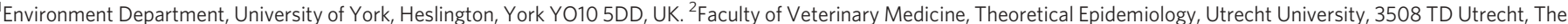
Netherlands. ${ }^{3}$ Spatial Ecology Department, Centre for Estuarine and Marine Ecology, Netherlands Institute of Ecology (NIOO-KNAW), 4400 AC Yerseke, The Netherlands.

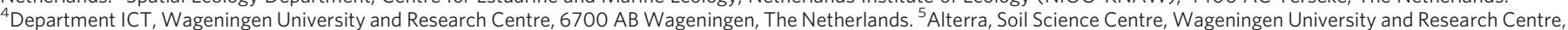
6700 AA Wageningen, The Netherlands. ${ }^{6}$ Nature Conservation and Plant Ecology Group, Wageningen University, 6708 PB Wageningen, The Netherlands. ${ }^{7}$ Department of

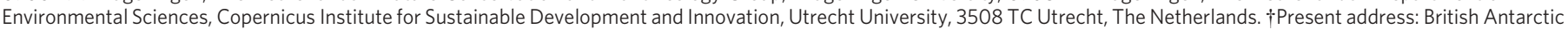
Survey, High Cross, Madingley Road, Cambridge CB3 OET, UK. 

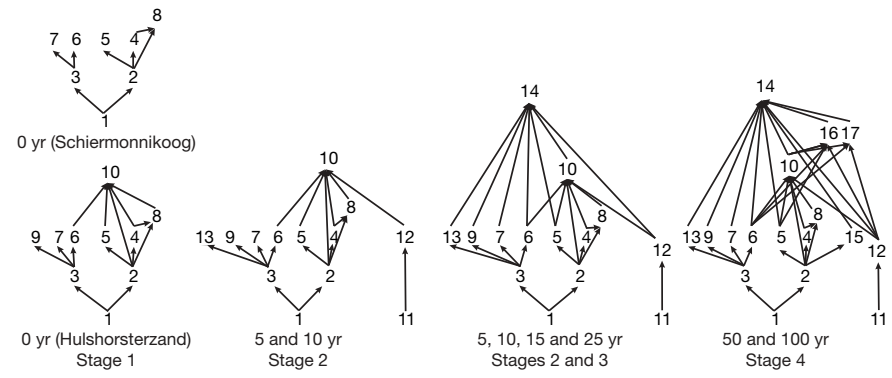

Figure 1 | Observed connectedness structures of the below-ground food webs of Schiermonnikoog and Hulshorsterzand, representative of the four successional stages. The numbers refer to the trophic groups: 1 , detritus; 2 , bacteria; 3 , fungi; 4 , flagellates; 5 , bacterivorous nematodes; 6 ,

fungivorous nematodes; 7 , noncryptostigmatic mites; 8 , amoebae; 9 , cryptostigmatic mites; 10 , predatory nematodes; 11 , roots; 12 , phytophagous nematodes; 13 , collembolans; 14 , predatory mites; 15 , bacterivorous mites; 16, nematophagous mites; and 17, predatory collembolans. Years refer to soil age (see Methods). For details and exceptions, see Supplementary Table 1.

predator-prey interactions: a measure of link density $(n C)$ and an analogue of May's complexity measure ${ }^{1} a(n C)^{\frac{1}{2}}$, where $a$ is the average interaction strength (see Methods). Then we related stability to a measure of food-web structure that incorporates the organization of interaction strengths: the maximum weight (see Methods) of omnivorous loops ${ }^{7}$. The measure does not include the shortest feedback loops, the predator-prey loops of two links (generating negative feedback), because they do not contribute to instability in these systems $\mathrm{s}^{7,18}$.

We found no strong and consistent relationship between link density $n C$ and stability in the two series (Fig. 2a). Incorporating the average strengths of the interactions did not explain the stability of the food webs either; there was also no consistent relationship between the analogue of May's complexity measure $a(n C)^{\frac{1}{2}}$ and stability (Fig. 2b).

We calculated the weights of the short two-link feedback loops and of omnivorous loops in the food webs (see Methods). In all the webs, loop weights decreased with loop lengths; the maximum weight of omnivorous loops in all food webs was that of loops with a length of three, with two top-down effects and one bottom-up effect. This was in correspondence with the results reported in ref. 7 . This is a positive feedback loop, because it consists of two negative effects and one positive effect.

We found that the maximum weight of omnivorous loops was strongly related to food-web stability in both series (Fig. 2c). A sensitivity analysis carried out with respect to observed biomass densities and to our way of deriving stability revealed the robustness of these results (see Supplementary Figs 1, 2 and 3).

The observed relationship between stability and the maximum weight of omnivorous loops does not depend on the specific foodweb types and configurations or on the range of complexity observed in these webs. This was illustrated by modelling the large set of food webs assembled in ref. 19 (Fig. 3; for parameterization, see Methods). We chose this set-acknowledging that it does not provide the most detailed data on real webs available - not to assess the actual stability of these food webs, but to test our relationship in a large series of webs from different environments, and with a wide variety of architectures.

On the basis of these results, we propose that stability in predatorprey communities (with at least one three-link loop) is limited by the maximum weight of (positive feedback) loops of length three, in biological terms described by:

$$
\max _{E_{3}}\left\{\left(\frac{f_{23}}{d_{3}} \frac{f_{12}}{d_{2}} e_{13} \frac{f_{13}}{d_{1}} \frac{B_{3}}{B_{1}}\right)^{\frac{1}{3}}\right\}
$$
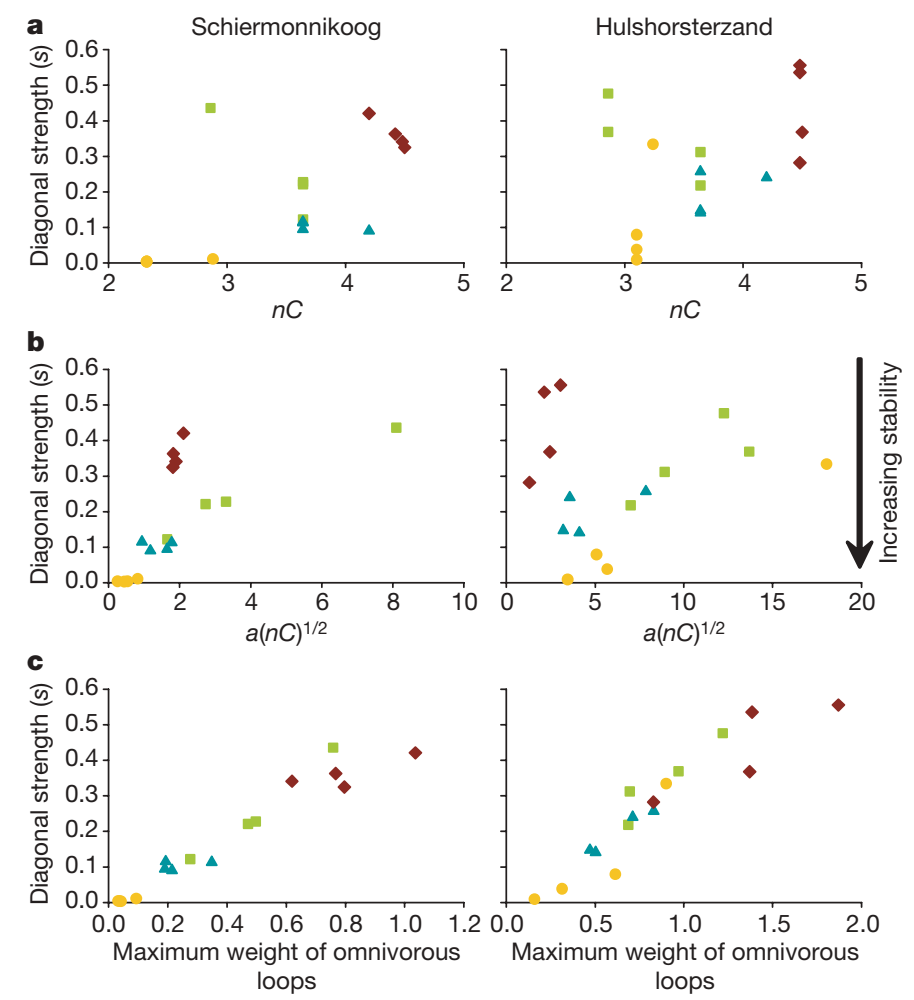

Figure 2 | Food-web stability related to measures of food-web structure, of Schiermonnikoog and Hulshorsterzand. a, Link density $n C$; b, $a(n C)^{\frac{1}{2}}$; c, maximum weight of omnivorous loops. The successional stages (1, yellow circles; 2 , green squares; 3 , blue triangles; and 4, brown diamonds) were each represented by four food-web replications. All quantities are dimensionless. For details, see Methods. Linear regression: $\mathrm{a}, y=-0.24+0.12 x, r^{2}=0.34$, $P=0.018$ (Schiermonnikoog) and $y=-0.16+0.12 x, r^{2}=0.19, P=0.096$

(Hulshorsterzand); b, $y=0.07+0.06 x, r^{2}=0.44, P=0.005$

(Schiermonnikoog) and $y=0.23+0.006 x, r^{2}=0.03, P=0.497$

(Hulshorsterzand); and c, $y=-0.007+0.47 x, r^{2}=0.94, P<0.001$

(Schiermonnikoog) and $y=-0.03+0.35 x, r^{2}=0.87, P<0.001$

(Hulshorsterzand). The sample size is $N=16$ in all statistical analyses.

where $E_{3}$ is the set of omnivorous loops of length three; the subscripts 1,2 , and 3 refer to bottom prey, intermediate predator and top predator in the loop, respectively; $f_{i j}$ is a mass-specific predation rate

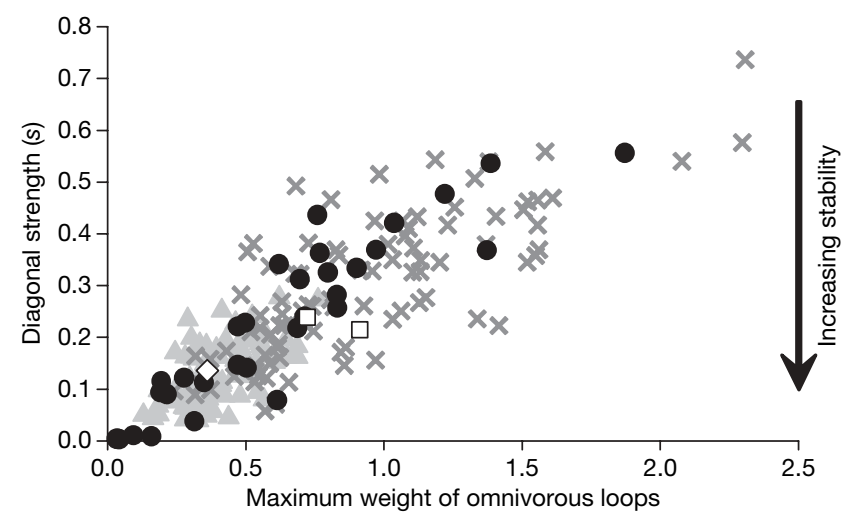

Figure 3 | Relationship between loop weight and stability for 'Cohen webs' compared with observed webs (black circles). Cohen webs were modelled with 10-fold (grey crosses) and 20 -fold (grey triangles) biomass decrease per trophic level (Methods). In 187 of 190 webs studied, the loop with maximum weight had length 3 (with 2 top-down effects and 1 bottom-up effect); in 3 it had length 4 ( 2 with 10 -fold (white squares) and 1 with 20 -fold biomass decrease (white diamond)). $n C$ ranged from 2.3 to 8.8 , and $n$ ranged from 14 to 99. Linear regressions: $y=0.03+0.28 x, r^{2}=0.71, N=190, P<0.001$ (Cohen webs); $y=0.01+0.34 x, r^{2}=0.85, N=32, P<0.001$ (observed webs). 
of predator $j$ on its prey $i$ in equilibrium; $e_{i j}$ is the efficiency with which $j$ converts food into biomass, from feeding on $i, 0<e_{i j}<1 ; d_{i}$ is the total natural (that is, non-predatory) specific death rate of $i$ in equilibrium (a scaling factor, used for consistency with the stability metric, which is also relative to these death rates, see Methods); and $B_{j}$ is the equilibrium biomass of predator $j$.

Expression (1) gives a clear insight into how organism traits and biomass structure affect loop weight and, hence, stability. Low topbottom biomass ratios $\left(\frac{B_{3}}{B_{1}}\right)$ contribute to low loop weight, as does skewed feeding of the top predator on intermediate and bottom prey $\left(f_{23} f_{13}\right)$. The latter effect could, for example, be enhanced by low biomass ratios of intermediate and bottom prey if the omnivores feed according to prey biomass.

We identified the predator-prey biomass ratios on which expression (1) directly depended for all the observed food webs (Supplementary Information). We found that the lower these predator-prey ratios were, the more stable the webs (Fig. 4). Moreover, in 21 of the 32 webs it was the same loop that had maximum (omnivorous) weight: that of predatory nematodes, bacterivorous nematodes and bacteria (crossed circles, Fig. 4). This showed how a single predatorprey biomass ratio had a key role in governing stability along the gradients. It also revealed a pattern over time in both gradients: this biomass ratio increased from the first to the second stage, when predatory nematodes were the top predators, and decreased again with complexity in the later stages, when predatory nematodes were no longer the top predators (Supplementary Fig. 4). In the last stage, this ratio was relatively low (Supplementary Fig. 4), but now loops higher in the web became the heaviest and new biomass ratios determined stability (Fig. 4).

The results suggest an alternating pattern of decreasing and increasing stability in the course of ecosystem succession: biomass is being built up on the top trophic level, causing an increase in maximum loop weight and a decrease in stability. New structure is created by the entrance of a new top predator. This causes predation pressure of the underlying trophic level on lower prey to decrease, which, in turn, decreases maximum loop weight and increases stability. Then, a further build-up of biomass on the highest trophic levels makes a new omnivorous loop the heaviest, increasing maximum loop weight and decreasing stability again. The observed

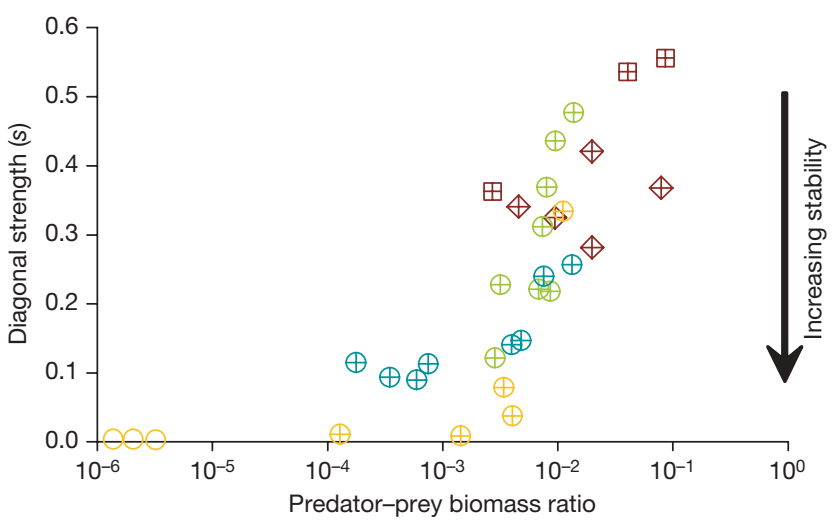

Figure 4 | Relationship between predator-prey biomass ratios and stability in the food webs along both successional gradients (Schiermonnikoog and Hulshorsterzand combined). Predator-prey biomass ratios are defined as the biomass ratios in a food web directly determining expression (1) (that is, determining interaction strengths in the loop with maximum omnivorous weight). These were $\frac{B_{10}}{B_{2}}$ (crossed circles), $\frac{B_{4} B_{8}}{\left(\sum_{i \in \Delta_{8}} B_{i}\right)^{2}}$ (open circles), $\frac{B_{10} B_{16}}{\left(\sum_{i \in A_{16}} B_{i}\right)^{2}}$ (crossed diamonds) and $\frac{B_{10} B_{17}}{\left(\sum_{i \in A_{17}} B_{i}\right)^{2}}$ (crossed squares), where $\Delta_{j}$ is the set of indices referring to the prey species of $j$, and subscripts refer to the trophic group (see Fig. 1). The colours refer to successional stage, as in Fig. 2. Linear regression of the relationship between $\frac{B_{10}}{B_{2}}$ and stability: $y=0.05+26 x, r^{2}=0.71, N=21, P<0.001$. pattern seems to reveal a cascading effect of increasing food-chain length with increasing productivity, as predicted in ref. 10. It suggests that top-down control operates not only within relatively simple food chains, but also within complex networks of intertwined chains ${ }^{20,21}$. Furthermore, this top-down control may have a key role in community assembly: the biomass build-up over succession does not lead to instability because predation is dampened again by other, higher trophic-level predators, thus solving May's paradox.

The emergence and persistence of biomass pyramids over succession has a clear basis in observation and theory ${ }^{10,11,22,23}$ and should be a general feature of most natural systems. Our results indicate that the shape of the biomass pyramid may have a crucial role in food-web stability. They suggest that more biomass at the top of food chains will limit stability through the weight of three-link (positive) feedback loops. Our simple metric emphasizes the importance of interaction strengths in the smallest omnivorous structures ${ }^{3,24}$.

In ecological networks, stability depends on the relationship between the positive and negative feedback loops, on one hand, and the self-damping within populations, on the other. By quantifying the feedback loops, our analysis takes a step in unravelling this relationship between interspecific and intraspecific forces. It is generally known that positive feedback loops are destabilizing and negative feedback loops are stabilizing. Our results suggest that the Achilles heel of a predator-prey network is its heaviest positive feedback loop. Thus, the approach may be of value for a broader class of ecological networks, in which the interactions are known to form skewed patterns of strong and weak links ${ }^{25,26}$.

\section{METHODS SUMMARY}

We took soil samples in the upper soil layers of each of the four stages in both vegetation successions in four replications. Soil samples were taken three times within a year (resulting in a total of 96 soil samples) to establish yearly average biomass densities.

We used the yearly averages of the biomass densities to calculate the interaction strengths-elements of the (jacobian) community matrices ${ }^{1}$ representing the soil food webs-using the methods from refs. 2 and 17. To do this, we regarded observed yearly average biomass densities as steady states-that is, states in which biomass growth rates of each population (gain through feeding) equal death rates (loss through natural death and predation). Effects of predators on their prey (negative interaction strengths) were mass-specific predation rates at steady state, and effects of prey on their predator (positive interaction strengths) were growth rates, per prey biomass, at steady state ${ }^{2,4,7,13}$. We calculated steady-state predation rates by using empirical values of total-mass-specific natural death rates, conversion efficiencies and prey preferences (rough estimations of prey's proportions in a predator's diet, relative to prey abundance).

We determined stability of the soil food webs using the method from ref. 7. Stability was calculated by assessing the level of intraspecific interaction (diagonal strength) needed for all eigenvalues in a community matrix to have negative real parts. This level of intraspecific interaction was expressed as the proportion of total-mass-specific natural (that is, non-predatory) death at the steady state that was density-dependent. Food webs that required less diagonal strength were called 'more stable'.

For the comparison of stability with measures of food-web structure, we calculated average interaction strength and loop weights ${ }^{7}$. Both quantities were scaled for total-mass-specific natural death rates.

Full Methods and any associated references are available in the online version of the paper at www.nature.com/nature.

\section{Received 20 April; accepted 7 August 2007.}

1. May, R. M. Will a large complex system be stable? Nature 238, 413-414 (1972).

2. de Ruiter, P. C., Neutel, A. M. \& Moore, J. C. Energetics, patterns of interaction strengths, and stability in real ecosystems. Science 269, 1257-1260 (1995).

3. McCann, K., Hastings, A. G. \& Huxel, R. Weak trophic interactions and the balance of nature. Nature 395, 794-798 (1998)

4. Rooney, N., McCann, K., Gellner, G. \& Moore, J. C. Structural asymmetry and the stability of diverse food webs. Nature 442, 265-269 (2006).

5. Olff, H., Huisman, J. \& van Tooren, B. F. Species dynamics and nutrient accumulation during early primary succession in coastal sand dunes. J. Ecol. 81, 693-702 (1993).

6. Berendse, F. \& Elberse, W. Th. in Perspectives on Plant Competition (eds Grace, J. B. \& Tilman, D.) 94-116 (Academic, New York, 1990). 
7. Neutel, A. M., Heesterbeek, J. A. P. \& de Ruiter, P. C. Stability in real food webs: weak links in long loops. Science 296, 1120-1123 (2002).

8. Grime, J. P. Plant Strategies and Vegetation Processes (Wiley, Chichester, 1979).

9. Lindeman, R. L. The trophic-dynamic aspect of ecology. Ecology 23, 399-418 (1942).

10. Oksanen, L., Fretwell, S. D., Arruda, J. \& Niemelä, P. Exploitation ecosystems in gradients of primary productivity. Am. Nat. 118, 240-261 (1981).

11. Kaunzinger, C. M. K. \& Morin, P. J. Productivity controls food-chain properties in microbial communities. Nature 395, 495-497 (1998)

12. Post, D. M., Pace, M. L. \& Hairston, N. G. Ecosystem size determines food-chain length in lakes. Nature 405, 1047-1049 (2000).

13. Pimm, S. L. \& Lawton, J. H. Number of trophic levels in ecological communities. Nature 268, 329-331 (1977).

14. Moore, J. C., Walter, D. E. \& Hunt, H. W. Arthropod regulation of micro- and mesobiota in belowground food webs. Annu. Rev. Entomol. 33, 419-439 (1988).

15. de Ruiter, P. C., van Veen, J. A., Moore, J. C., Brussaard, L. \& Hunt, H. W. Calculation of nitrogen mineralisation in soil food webs. Plant Soil 157, 263-273 (1993).

16. Gardner, M. R. \& Asby, W. R. Connectance of large dynamic (cybernetic) systems: critical values for stability. Nature 228, 784 (1970).

17. Hunt, H. W. et al. The detrital food web in a shortgrass prairie. Biol. Fertil. Soils 3 , 57-68 (1987).

18. Hofbauer, J. \& Sigmund, K. in The Theory of Evolution and Dynamical Systems 204-206 (Cambridge Univ. Press, Cambridge, 1988)

19. Cohen, J. E., Briand, F. \& Newman, C. M. Community Food Webs: Data and Theory (Springer, Berlin, 1990).
20. Halaj, J. \& Wise, D. T. Terrestrial trophic cascades: how much do they trickle? Am Nat. 157, 262-281 (2001)

21. Finke, D. L. \& Denno, R. F. Predator diversity dampens trophic cascades. Nature 429, 407-410 (2004)

22. Hairston, N. G. Sr, Smith, F. E. \& Slobodkin, L. B. Community structure, population control, and competition. Am. Nat. 142, 379-411 (1960).

23. Moore, J. C., de Ruiter, P. C. \& Hunt, H. W. Influence of productivity on the stability of real and model ecosystems. Science 261, 906-908 (1993).

24. Holt, R. D. \& Polis, G. A. A theoretical framework for intraguild predation. Am. Nat. 149, 745-764 (1997).

25. Scheffer, M. \& van Nes, E. H. Self-organized similarity, the evolutionary emergence of groups of similar species. Proc. Natl Acad. Sci. USA 103, 6230-6235 (2006).

26. Bascompte, J., Jordano, P. \& Olesen, J. M. Asymmetric coevolutionary networks facilitate biodiversity maintenance. Science 312, 431-433 (2006).

Supplementary Information is linked to the online version of the paper at www.nature.com/nature.

Acknowledgements We thank E. Biewenga, P. Bolhuis, B. van der Boom, K. Kampen, $M$. Veninga and W. Willems for assistance in collecting and analysing the soil samples. We thank S. Burgers, J. Krumins and P. Morin for comments on the manuscript.

Author Information Reprints and permissions information is available at www.nature.com/reprints. Correspondence and requests for materials should be addressed to A.-M.N. (anjute@bas.ac.uk). 


\section{METHODS}

Soil layers and methods of sampling. Soil layers in the four developmental stages were: 1 , bare soil, mineral layer (C horizon); 2 , sparse grasses, mineral layer (C horizon); 3 , dense herbaceous vegetation, humus-rich layer (A horizon) of $2-5 \mathrm{~cm}$; and 4 , woody vegetation with undergrowth, organic litter layer (F horizon) of more than $5 \mathrm{~cm}$. Dominating plant species in the above-ground vegetation along the gradient of Schiermonnikoog were: 2, Festuca rubra; 3 , Ammophila arenaria and F. rubra; 4, Sambucus nigra and Urtica dioica. Dominating plant species in the above-ground vegetation along the gradient of Hulshorsterzand were: 2, bryophytes with dispersed Corynephorus canescens; 3, mixed vegetation of bryophytes, lichens, C. canescens and young Pinus sylvestris; 4, P. sylvestris forest with an understory of Deschampsia flexuosa and bryophytes. The ages of the stages in terms of soil development were approximately 0 , 10,25 and $100 \mathrm{yr}$ in Schiermonnikoog and 0, 5, 15 and $50 \mathrm{yr}$ in Hulshorsterzand.

In each of the four stages in both successions, soil samples were taken three times within a year, in four replications, to establish yearly averages: in November 1993, March 1994 and August 1994 in Schiermonnikoog, and in October 1993, March 1994 and September 1994 in Hulshorsterzand. A total of 96 soil samples were processed as described in refs 15, 27 and 28. Biomass of bacteria was determined using the method from ref. 29, of fungi using ref. 28 , and of protozoa, nematodes and microarthropods using ref. 27. Yearly average biomass densities of all functional groups were determined for 32 food webs (Supplementary Information). All measurements for a single food-web replicate were taken from the same soil sample; that is, they represented replications of entire food webs.

Earlier studies on the above-ground vegetation in both series indicate that above-ground productivity increases with age in these stages ${ }^{5,6}$. Observed overall soil nitrogen mineralization rates indicated a similar increase in below-ground productivity along both gradients: 1.9 (1.1-2.9), 7.9 (6.2-9.1), 39 (35-46) and 80 (70-92) $\mathrm{kg} \mathrm{N} \mathrm{ha}^{-1} \mathrm{yr}^{-1} \mathrm{~cm} \mathrm{depth}^{-1}$ in Schiermonnikoog, and 1.1 (0.49-2.5), 7.9 (5.3-11.4), 40 (34-48) and $86(49-160) \mathrm{kg} \mathrm{Nha}^{-1} \mathrm{yr}^{-1} \mathrm{~cm} \mathrm{depth}^{-1}$ in Hulshorsterzand.

Calculation of interaction strengths. Community matrices were derived using the method from ref. 1. The community matrix $A$ is the jacobian matrix of interaction obtained by linearizing the nonlinear dynamical systems of growth equations and evaluated at the equilibrium with all groups present ${ }^{1}$. The elements of $A$, partial derivatives of the growth equations, are the interaction strengths $\alpha_{i j}$, representing the effects of the trophic groups on each other near equilibrium. Assuming direct dependence of feeding rates on predator population density, effects of predator $j$ on prey $i$ are defined as predation per predator biomass in equilibrium: $\alpha_{i j}=-f_{i j}$, where $f_{i j}$ is a mass-specific predation rate of predator $j$ on its prey $i$ in equilibrium ${ }^{2,4,7,13}$. Assuming also direct dependence of feeding rates on prey population density, effects of prey on their predator are defined as predator growth resulting from this predation, per prey biomass in equilibrium. This means that effect sizes of prey $i$ on their predator $j$ were related to effects of the predator on the prey according to: $\alpha_{j i}=-e_{i j} \frac{B_{j}}{B_{i}} \alpha_{i j}$, where $B_{j}$ is the equilibrium biomass of predator $j$ and $e_{i j}$ is the efficiency with which $j$ converts food into biomass, from feeding on $i, 0<e_{i j}<1$ (refs $2,4,7,13$ ). The same analysis can be performed for descriptions of interaction that incorporate more complex functional responses. This does not change the effects of predators on prey and will not fundamentally change the relationship with effects of prey on predators; it will generally, at most, introduce another stabilizing factor. Thus we were able to concentrate on the simple case outlined above without clouding the issue by more complicated formulations, for which we had little empirical information.

Values of interaction strengths of all the interactions in the soil food webs were calculated using the method of ref. 2. This involved the following procedure. Mass-specific predation rates were derived from observed average biomass densities using the steady state assumption, following the method from ref. 17: $\sum_{i \in A_{j}} f_{i j} B_{j}=\frac{d_{j} B_{j}+\sum_{k \in \Gamma_{j}} f_{j k} B_{k}}{e_{j}}$, where $\Delta_{j}$ is the set of indices referring to the prey species of $j, \Gamma_{j}$ is the set of indices referring to the predator species of $j, B_{j}$ is the observed yearly average population density of the predator, $d_{j}$ is the mass-specific natural death rate (per yr), $e_{j}$ is a conversion efficiency, $0<e_{i j}<1$, making the simplifying assumption $e_{i j}=e_{j}$ for all $i \in \Delta_{j}$, and using $f_{i j}=\frac{p_{i j} B_{i}}{\sum_{h \in A_{j}} p_{h j} B_{h}} f_{j}$, where $f_{j}=\sum_{i \in \Delta_{j}} f_{i j}$ and $p_{i j}$ is a prey preference factor. Preference factors were published values on the basis of experiments involving feeding trials and gut content analyses in relation to prey availability ${ }^{17}$. The values for these preferences are rough estimates, but they give approximations in terms of orders of magnitude. All parameters are defined as positive.
The diagonal terms in a community matrix referring to self-damping (intraspecific competition) of the organisms were determined on the basis of the empirical values of natural death rates, representing all non-predatory losses that can be expected in populations in their natural environment ${ }^{17}$. Using the method from ref. 2 they were modelled as $\alpha_{i i}=-s d_{i}$, where $d_{i}$ is the total natural specific death rate of $i$ in equilibrium (defined positive), and $s$ is the proportion of natural death in equilibrium (that is, assumed equal for all species in a food web) that is attributed to density-dependent mechanisms $(0<s<1$; for a detailed argument, see Supplementary Information). The value of $s$ was unspecified and was derived to assess stability (see below).

Calculation of stability. To allow for variation in the matrix element values, 100 community matrices were used to represent each web, drawing off-diagonal element values randomly from uniform intervals around the calculated values $\left\langle 0,2 \alpha_{i j}\right\rangle, i \neq j$ using the method from ref. 2. Departing from ref. 2 and using the method from ref. 7, diagonal elements $\alpha_{i i}=-s d_{i}$ were not set at certain fixed, arbitrary levels, but were varied. The procedure is as follows: matrix stability was determined in the standard way, that is, by calculating the eigenvalues; if all eigenvalues have negative real parts, a matrix is stable. Values for $s$ were assessed as the minimum value that was necessary for matrix stability (for details of the procedure, see Supplementary Information). Stability of a food web was then calculated as the mean value of $s$ of the 100 sampled community matrices. This provided a robust measure of stability. For further explanation and motivation, see Supplementary Information.

Owing to a lack of detailed biological information, we have chosen the selfdamping factor $s$ to be the same for all species and to be constant during successional development. We acknowledge that the levels of intra-specific interaction could vary between the species in a food web and change over succession for independent reasons. Also, we note that stability was not measured directly by, for example, perturbing food webs experimentally. It remains to be determined how good a surrogate our measure is. However, it should be noted that such experiments would be difficult because 'stability' is not a directly measurable variable and, even with experiments, surrogate quantities would have to be used.

Calculation of average interaction strength. Average interaction strengths were calculated:

$$
a=\frac{\left\{\left|\sum_{j \in M} \sum_{i \in \Delta_{j}} \alpha_{i j}\right|\left(\sum_{j \in M} \sum_{i \in \Delta_{j}} \alpha_{j i}\right)\right\}^{\frac{1}{2}}}{L\left(d_{1} \ldots d_{n}\right)^{\frac{1}{n}}}
$$

where $L$ is the total number of feeding links, $M$ is the set of indices referring to all predator species, $\Delta_{j}$ is the set of indices referring to the prey species of $j$, and $d_{i}-$ the total natural specific death rate of $i$ in equilibrium (defined positive)-is a scaling factor used to make this metric comparable with the stability metric, which is also relative to these death rates. The geometric means of negative topdown and positive bottom-up effects were taken separately because negative and positive interaction strengths differed systematically by about two orders of magnitude ${ }^{2}$. The precise way in which a mean was taken had no effect on the resulting patterns.

Calculation of loop weights. Loop weight is defined as the geometric mean of the absolute values of the jacobian elements in a loop ${ }^{7}$ :

$$
w^{(k)}=\left|\frac{\alpha_{i_{1} i_{2}} \alpha_{i_{2} i_{3}} \ldots \alpha_{i_{k} i_{1}}}{d_{i_{1}} d_{i_{2}} \cdots d_{i_{k}}}\right|^{1 / k}
$$

where $w^{(k)}$ is the scaled weight of a loop of length $k$. There can be different loops of length $k$, with consequently different loop weights.

Parameterization of 'Cohen webs'. Community matrices were derived for 95 food webs assembled in ref. 19 ('Cohen webs') from hypothetical biomass structures of a 10 -fold and 20-fold biomass decrease per trophic level, using the method from ref. 7. They were parameterized with equal values for all groups, assuming $e_{i j}=e=0.1, d_{j}=d=1$, and no specific prey preferences.

27. Brussaard, L., Bouwman, L. A., Geurs, M., Hassink, J. \& Zwart, K. B. Biomass, composition and temporal dynamics of soil organisms of a silt loam soil under conventional and integrated management. Neth. J. Agr. Sci. 38, 283-302 (1990).

28. Hassink, J., Lebbink, G. \& van Veen, J. A. Microbial biomass and activity of a reclaimed polder soil under a conventional or a reduced-input farming system. Soil Biol. Biochem. 23, 515-524 (1991).

29. Bloem, J., Veninga, M. \& Shepherd, J. Fully automatic determination of soil bacterium numbers, cell volumes, and frequencies of dividing cells by confocal laser scanning microscopy and image analysis. Appl. Environ. Microb. 61, 926-936 (1995) 